\title{
COMMISSION 33: STRUCTURE AND DYNAMICS OF THE GALACTIC SYSTEM (STRUCTURE ET DYNAMIQUE DU SYSTÈME GALACTIQUE)
}

\author{
Report of Meetings, 20, 21, 22 and 26 August 1970
}

President: G. Contopoulos.

SECRETARY: F. J. Kerr.

\section{Business Session}

Following a request to all Commissions by the Executive Committee, a set of by-laws was prepared by the Organizing Committee and adopted by the Commission.

1. A member of the Organizing Committee serves for two consecutive terms and then retires unless he becomes President or Vice-President. A retiring President stays one more term as member of the Organizing Committee. Any retiring member can be reelected at a later time.

2. The President serves for one term and is then normally succeeded by the Vice-President. Proposals for the new Vice-President and new members of the Organizing Committee to replace those retiring are made by the Organizing Committee at the first meeting of Commission 33 during a General Assembly. If any member of the Commission proposes other names, a secret ballot is taken of all members of the Commission present.

3. New members of Commission 33 are proposed by at least one member of the Commission. The Organizing Committee decides on each proposal.

The proposed names of Commission officers were accepted.

During the last triennium, the Commission report in the IAU Transactions was severely reduced in size because of financial difficulties. However, the Commission produced on its own initiative a longer report which appeared as a publication of the University of Thessaloniki. Opinions were sought on the value of this dual form. After several very favourable comments it was agreed that two reports should again be attempted, in spite of the extra work involved.

The following resolution was proposed by the Organizing Committee:

We propose that the superscript II be omitted from the new galactic coordinates (those adopted by the IAU in 1958); henceforth they should be simply $l$ and $b$. We recommend that authors adopting this practice should indicate in the beginning of each paper that $l$ and $b$ are the new galactic coordinates. The old coordinates should retain the superscript $I$.

Ogorodnikov said he was not against the proposal, but emphasized that it should be regarded as an exceptional case, as changes are always confusing. Bok pointed out that the change is being carried out slowly, three years having elapsed since an earlier motion to the same effect. Blaauw stated that a major reason for the proposal was to recognize a trend already in effect.

The resolution was carried.

Contopoulos pointed out that the Selected Areas Committee has worked for many years - in fact, its field of work was originally the central subject of the Commission. He then proposed that there should no longer be a separate committee, but instead its work should be taken over by the full Commission. Elvius had been included in the new Organizing Committee to facilitate this change.

The meeting approved the dissolution of the Committee, adding a vote of thanks to the Committee and in particular to Elvius for their very good work over the years.

\section{PAPERS}

J. Einasto: A Proposal for Galactic Research.

K. Lodén: The Stockholm Survey of the Southern Milky Way.

J. D. Wray and B. E. Westerlund: An Atlas of the Southern Milky Way. 
C. B. Stephenson and N. Sanduleak: The Cleveland Southern O-B Star Survey.

J. A. Agekjan: The Third Integral of Motion in a Stationary Stellar System.

J. Einasto: On the Structure and Evolution of the Galaxy.

\author{
Second Meeting \\ RR LYRAE STARS
}

Joint Meeting with Commissions 27, 24, 30, 32 and 37.

R. v.d. R. Woolley: On the Determination of the Absolute Magnitudes of the RR Lyrae Stars.

R. F. Christy: Absolute Magnitudes of RR Lyrae Stars.

G. van Herk: Review of Observational Data on RR Lyrae Stars.

S. V. M. Clube: Absolute Magnitudes of RR Lyrae Variables.

A. R. Klemola: The Lick Observatory Program on Proper Motions of RR Lyrae Stars.

Third Meeting

SPIRAL STRUCTURE AND RELATED PROBLEMS

M. Lecar: Report on the IAU Colloquium No. 10 'Gravitational $n$-Body Problem'.

F. H. Shu: Observational versus Theoretical Spiral Patterns in External Galaxies and in our Galaxy.

C. Yuan: On the Kinematics of Nearby Stars.

A. Kalnajs: Non-Linear Effects in Axisymmetric Waves.

F. J. Kerr: Local Spiral Structure.

W. Iwanowska: A Statistical Approach to the Problem of Stellar Populations.

L. Martinet and A. Hayli: On the Dynamics of the RR Lyrae Variables and the Existence of a Third Integral of Motion in the Galaxy.

\title{
Fourth Meeting
}

THE DISTANCE SCALE OF OUR GALAXY

B. J. Bok: Report on the RR Lyrae Meeting.

O. J. Eggen: The Distance of the Hyades.

P. A. Wayman: The Error Limits in the Convergence Method for Determining the Distance of the Hyades.

A. Behr: The Ratio of Interstellar Extinction to Reddening.

J. D. Fernie: The General Consequences of Uncertainty in the Ratio of Total to Selective Interstellar Absorption.

J. H. Oort: Summary and Conclusions.

Fifth Meeting

SELECTED AREAS

Chairman: T. Elvius.

SECRETARY: K. Lodén.

There was no discussion about the printed reports (full version in Contr. astr. Dep. Univ. Thessaloniki 53, 111, 1970; short version in Transactions IAU 14A, 383, 1970), which were accepted. 
At the business meeting of Commission 33 it had been decided to dissolve the special Committee for Selected Areas and to include the Chairman in the Organizing Committee of the Commission, which should report directly on Selected Area matters.

Kerr reported on a 21-cm hydrogen-line survey of all the Selected Areas. Profiles have been observed for the central point of each field and at points north, south, east, and west of this point, stepped off $21^{\prime}$. The observations for Selected Areas 1-163 were made at Green Bank with the NRAO 140-ft telescope and the 413-channel receiver. The velocity resolutions are 0.69 and 2.78 $\mathrm{km} / \mathrm{sec}$. A catalogue of calibrated profiles will be produced as a first result, and the reduction is under way. Results for individual areas can be sent to those who are interested. Future work will include studies of the velocity field and comparisons of radio and optical data. The southern Selected Areas were observed at Pereyra, Argentina.

Ardeberg reported on the following work performed at La Silla, Chile, in Selected Areas 165, 188, and 205:

1. SA 165: Photoelectric UBV photometry of all stars brighter than $V=10.5$ (and some fainter) and of all stars of spectral types earlier than $G 0$ and with $V \leqslant 12.5$; photographic UBV photometry for all stars brighter than $V=15$; photoelectric polarimetry for the 20 brightest stars; slit spectra (dispersion $70 \AA / \mathrm{mm}$ ) for some bright stars; objective prism spectra (dispersion $110 \AA / \mathrm{mm}$ ).

2. SA 188: The same photoelectric photometry as in SA 165; objective prism spectra obtained.

3. SA 205: Photoelectric photometry for all stars brighter than $V=10.5$; photoelectric polarimetry for the 20 brightest stars; objective prism spectra obtained.

Reductions are now under way.

Griffin has measured radial velocities with a photoelectric technique for about 500 stars in Selected Areas 68 to $91\left(+15^{\circ}\right.$ declination zone). The stars range from G5 to M in spectral type and from 7 to 10 in $V$. The standard error is about $1 \mathrm{~km} / \mathrm{sec}$. The work is to be published in Monthly Notices.

Terzan spoke on his project of constructing maps for each of the 206 Selected Areas and of compiling a reference catalogue from all existing published catalogues and investigations, with keys to the numbering in the various catalogues of the stars considered. Terzan demonstrated maps for SA 68, derived by means of a computer programme due to Ounnas. The technical and financial problems were discussed. It was further mentioned that the long exposures of southern Selected Areas taken at the Uppsala Station at Mount Stromlo could be used for making photographic maps available. The possibility of using inexpensive overlays with printed numbers on them to be placed upon already existing charts was mentioned by Bok. Roman reported that she had maps available for a number of fields. Murray and Bok proposed that the data compiled by Terzan should be available on magnetic tape instead of being printed, copies of which could be obtained relatively cheaply by interested astronomers.

The chairman concluded this last Selected Areas meeting by thanking all who during a long series of years had contributed to the work of the Selected Areas Commission, Sub-Commission, and Committee.

A more extensive report of the Commission meetings, including abstracts of the paper, can be obtained from the secretary. 